\title{
Factors Influencing Practice of Human Resource Information System in Organizations: A Hybrid Approach of AHP and DEMATEL
}

\author{
Abdul Kadar Muhammad Masum ${ }^{1}$, Faisal Bin Abid $^{2}$ \\ ABM Yasir Arafat ${ }^{3}$ \\ Department of Computer Science and Engineering \\ International Islamic University Chittagong \\ Chittagong, Bangladesh
}

\author{
Loo-See Beh ${ }^{4}$ \\ Department of Administrative Studies and Politics \\ University of Malaya \\ Kuala Lumpur \\ Malaysia
}

\begin{abstract}
This paper blends the development of the Technology-Organization-Environment (TOE) framework and Human-Organization-Technology (HOT) fit model to identify the factors that influence the administration choice in embracing human resource information system (HRIS) in the organizations. Here, a hybrid Multi-Criteria Decision Making (MCDM) model combining the Decision Making Trial and Evaluation Laboratory (DEMATEL) and Analytic hierarchy Processes (AHP) is used to achieve the objective of the study. In this study, the experts agree that the staffs IT skill is most significant than other factors for the Human dimension. Similarly, IT infrastructure, top level support, and competitive pressure are the most vital factors for Technology, Organization and Environment dimensions respectively. Moreover, this paper will help the managers to take care of some factors that are vital for HRIS implementation in the organizations.
\end{abstract}

Keywords-Analytic Hierarchy Processes (AHP); Decision Making Trial and Evaluation Laboratory (DEMATEL); factor; Human Resource Information System (HRIS); Multi-Criteria Decision Making (MCDM) Model

\section{INTRODUCTION}

Conventional human resource management (HRM) processes have been moved to HRIS in order to achieve the organizational objectives [1]. Specialists realized the magnitude of HRIS applications and investigated an expansive number of persuasive elements for the choice and usage of HRIS among business organizations [2]. Experts contended that the relative weight of investigated factors might be changed alongside gradual development and its settings. Besides, researchers unveiled that the technological innovation is consistently unpredictable in a competitive setting. And, it is crucial to comprehend the possible factors impacting the choice of HRIS selection and usage in the associations for a specific setting [3].

The technology innovation adoption concept has evolved globally [4] though there are still some constraints for appropriateness in developing settings of innovation models of Western countries [5, 6]. In compared to the West, Bangladesh is incredibly unique considering innovation, cultural, economic conditions as a developing nation. Thus, investigating the applicability model for HRIS adoption in developing countries is imperative. Past research indicates that there is a research gap in connection to the potential factors influencing the use of HRIS adoption in developing nations [7]. Therefore, the prime research target of this study is to exploring the most important factors of HRIS adoption among organizations of Bangladesh. Following this goal, we have some novelties in this paper. Firstly, the current study suggests an IT adoption model in the context of Bangladesh as there is a lack of theories of IT adoption for developing nations. Secondly, this paper reveals a thorough investigation assessing the essential level of interdependency among basic variables for the selection of HRIS usage in developing nations. Moreover, this study proposes an MCDM combining the DEMATEL and AHP approaches to assess and discover the significance level of the determining elements for HRIS usage in Bangladesh.

The remaining part of the paper is organized as follows. Section II labels the factors of human resource information system adoption based on Human-Organization-Technology (HOT) fit model and technological, organizational, and environmental (TOE) model. The research model and research methods and techniques that are adopted to achieve the objectives of the study are presented in Section III. Section IV includes data analysis along with some interesting results. A brief discussion on findings is provided in Section $\mathrm{V}$, before concluding the paper in Section VI.

\section{LITERATURE REVIEW}

Researchers identified some factors for technological innovation in different settings. In this paper, we examined a few variables for IT innovation adoption. Both subjective and quantitative methodologies were utilized in technological adoption research. However, the subjective is a thoroughly detectable approach. It is apparent from exploring past researches that, the most significant and widely used fifteen variables are adopted dividing into four dimensions e.g. human (the characteristics of senior executives, Staffs IT skill), technology (comparative advantage, perceived compatibility, perceived complexity, and IT infrastructure), organization (top level support, organizational culture, organizational structure, organizational size, perceived cost), and environment (competitive pressure, support from technology service provider, government support)). The related research findings 
regarding the above variables are summarized below. Remarkably, in most past studies, HRIS and electronic human resource management (e-HRM) are used interchangeably [8].

In this study, the three variables of human dimension is characteristics of senior executives (innovativeness and IT knowledge), staffs IT skill, and employee Behavioral Characteristics. Adoption of a new system is an strategic decision of an organization [9]. Therefore, the decision towards adopting or rejecting an innovation is depends on personal attributes of top managers and skill on IT [10]. On the contrary, a couple of studies asserted that senior officials' IT learning and creativity does not influence IT adoption in organizations [11]. Previous research of Alam, Masum, Beh and Hong [5] revealed that organizations having staff with IT learning background create probability of IT enabled HRM applications. In line with previous research, Teo, Lim and Fedric [4] and Bian [12] showed IT ability and employee intention to use IT applications are strong indicators to choose HRIS in organizations of China. However, a few scholars found that IT abilities of staff was unimportant factor for technological innovations considering multiple settings [2, 13].

The organization dimensions include five variables such as top level support, organizational culture, organizational structure, organizational size, and perceived cost. Some researchers identified the support of top management as a vital factor for IT application use [2, 4]. Conversely, few studies on adoption of IT have claimed that the support from top level is not always a subject of influence to adopt IT in organization. $[11,14]$. Various scholars explored the variable Organizational culture to be an important factor for the selection of IT applications [15, 16]. In addition, Cooper and Schindler [17] resolved that incase of any clashes in information system (IS) of an organization the software is become abandoned or customized so that it matches the existing culture of business organizations. Conversely, some researchers argued that organizational culture is not an essential divider amongst adopters and non-adopters of modern IT application [18]. Moreover, earlier studies on 110 manufacturing firms in Singapore confirmed that organizational size is the only constantly accepted factor among the possible causes for HRIS use in the organizations [4]. Scholars contended that if expenses are perceived to be high, people will be less inclined to invest in the selection of HRIS [5]. A contemporary study on the adoption of internet business in Iranian SMEs uncovered that the budgetary viewpoint (high expenses) is the sole issue behind not embracing web-based business applications [19].

The four variables of technology dimension such as perceived compatibility, perceived complexity, comparative advantage, and IT infrastructure are widely established technological factors in IT innovation research. Teo, Lim and Fedric [4] indicated that choice of HRIS adaption is strongly connected to an amiable impression of HRIS in human resources (HR) department. Similarly, Al-Dmour [2] claimed the significance of perceived relative advantage on HRIS usage. Whereas perceived compatibility was evident to be highest influencer on the use of HRIS [4]. Likewise, Ghobakhloo, Arias-Aranda and Benitez-Amado [19] recommended perceived compatibility as key component of IT application in adoption stage. In this regards, researchers concluded that the successful adoption of new technology suffers from a high extent of uncertainty and risk due to perceived complexity For this reason Gutierrez, Boukrami and Lumsden [14] mentioned that, in the UK, perceived complexity has a strong influence in the implementation of distributed computing services. Conversely, Teo, Lim and Fedric [4] and Bian [12] found it as a non-discriminate factor among the firms. And, few researchers have claimed that compatibility does not influence innovation adoption[11]. In additions, researchers uncovered that perceived complexity is not a vital factor for HRIS or related IT appropriation choice in firms or organizations $[4,11]$. Recent studies indicated that IT infrastructure is the most common barrier to adopt IT enabled applications in organizations of the developing countries [1, 20]. Conversely, some current research showed that IT infrastructure is an insignificant factor for IT innovation adoption [5, 21].

Lastly, environmental dimension consist three variables like competitive pressure, support from technology service provider and government support. Ghobakhloo, Arias-Aranda and Benitez-Amado [19] recommended that competitive pressure is one of the most important variables taken into account for deciding to implement IT strategies within an organization. Additionally, Bian [12] discovered that pressure from competitor was a critical factor for using HRIS in China. In Jordanian firms, Al-Dmour [2] stated that the availability of IT providers and their sponsorship is the most worthy factor in an environmental context for using HRIS. Conversely, various studies on IT adoption have concluded that support from technology service provider is not identified to be a major factor [11, 22]. A present study on the adoption of HRIS in public organizations of Australia uncovered that the government rule and regulations is very important determinants for HRIS adoption and implementations [23]. Similarly, AlDmour [2] claimed that government policy regarding IT applications adoption is one of the significant factors for HRIS implementation in firms of Jordan.

In contemporary research on organizations of Bangladesh, researchers claimed that organization, environment and technology are observed to be the most persuasive dimensions for HRIS adoption [5]. The authors obtained five most critical factors for HRIS adoption in organizations. And, these factors are IT infrastructure, Staffs IT capabilities, top level support, perceived cost, and competitive pressure. Likewise, Masum [1] stated that the foremost obstruction for executing HRIS in developing countries is the lack of management knowledge, experience, training, and fear of expense. But, the advantages of the HRIS is outweigh the limitations [24]. The past studies indicate that research on HRIS in Bangladesh is in early-stage for Bangladesh setting [25].

Amalnick, Ansarinejad and Nargesi [26] adopted DEMATEL and ANP evaluated some important factor for successful implementation of ERP systems. They explored that ERP vendor selection, project team, project/business plan and business model, management, and budgeting made up the top five success factor for ERP implementation in the organizations. In a recent research, scholars employed AHP with Fuzzy AHP (FAHP) and group decision-making (GDM) 
to identify the diversified factors from different dimensions of the web-based E-Learning system in university context [27]. A study on an Iranian steel company, Rouhani, Ashrafi and Afshari [28] evaluated CSFs in ERP using a hybrid model based on fuzzy DEMATEL and fuzzy AHP. The results exposed that project champion, clear project plan, project team competence, training and education and organizational culture were among the most significant 8 aspects to be considered.

Interestingly, most of the past studies depicted descriptive statistics, absence of robust inferential statistics, and advanced artificial intelligence (AI) tools and techniques are not used. So, research gap is visible and that need be resolved by different types of AI techniques such as MCDM Model Approach. MCDM is one of the famous techniques to manage complex issues that show high vulnerability, conflicting goals, different interests and numerous points of view [29]. Moreover, MCDM approaches are viable in decision making, weighing and choosing the most fitting options.

\section{RESEARCH METHODS}

Technology-Organization-Environment (TOE) structure is the widely used model in technology adoption at organizations. The model consists of triple dimensions such as technological, organizational and environmental. But, the important limitation of this model is no consideration of human factors such as IT skill and innovative knowledge of managers and employees. Reversely, the latest model of IT application adoption named Human-Organization-Technology (HOT) fit at organizational level also suffers from the limitation to explaining the total modalities of IT enabled applications such as HRIS adoption. Particularly, the model not recognized external (environmental) factors though environmental factors greatly influence the organizational systems. So, theoretical gap is identified. Thus, the most relevant factors of HRIS adoption at the organizational level may significantly predict combining four dimensions (i.e., human, organizational, technological, and environmental) in context of Bangladesh.

Through extensive literature review, 15 factors were chosen. The factors are characteristics of senior executive (CHAR_SEC), staffs IT skill (SIS), Employee Behavioral Characteristics (EMPB_CHAR), Comparative Advantage (COM_ADV), Perceived Compatibility (PER_COMPA), Perceived Complexity (PER_COMPL), IT Infrastructure (IT_INFRAS), Top level support (TLS), Organizational Culture (OC), Organizational Structure (OST), Organizational Size (OSIZE), Perceived Cost (PER_COST), Competitive Pressure (CP), support from technology service provider (TVS), Government Support (GOV_SUP). A hybrid MCDM model is utilized to identify the interconnections of variables with the AHP and DEMATEL strategies.

Data was collected using questionnaire survey. And, the questionnaire attempted to obtain information relevant information related to the research such as factors of HRIS adoption decision, extent of usage HRIS application etc. We have selected 15 human resource managers from 15 reputed manufacturing organizations of Bangladesh, who have adequate knowledge and expertise on HRIS. In order to attain anonymity and free from biasness, the self-administered structured questionnaire was employed. For furtherance of the research the survey method was used as the sampled elements and the variables were treated having no to control, influence, or manipulate them.

AHP, one of the famous MCDM techniques is used to analyze complex decisions for broader application areas. In the literal context, DEMATEL is adjusted for various subjective and factor-related points including modern industrial arranging, basic leadership, feasible development and other world issues [30]. Realizing the benefits of hybrid MCDM especially integrating DEMATEL and AHP techniques, scholars used them in different settings such as product selection, supplier selection, software selection, and so on [31]. DEMATEL is a helpful strategy in order to analyze cause-effect connections between factors and dimensions. But DEMATEL itself can't decide the weights of individual criteria, where AHP proves to be useful. The two techniques offer help in managing complex issues, as decision producers can have a superior understanding of the issues to be unraveled. As indicated by Horng, Liu, Chou, Yin and Tsai [32], combining DEMATEL and AHP techniques altogether can give a supportive tool for recognizing the basic traits of policy arrangement usage as well as computing the weights of the business decision criteria. Thus, DEMATEL and AHP strategies are embraced to fulfill the goals of this study.

\section{RESULTS}

As we discussed in the previous section, a conceptual research strategy has been developed in this study. Consequently, in this segment, we constructed a combination of MCDM models for the procedure of HRIS adoption selection. The proposed MCDM model includes two principle stages namely AHP and DEMATEL.

\section{A. Analytic Hierarchy Process (AHP)}

Based on the inputs, taken from the respondents, AHP is used to derive ratio scales from pairwise assessments. The consistency ratio was the parameter used for finding out the accuracy of the respondents. In case of being inconsistent, the respondents were told to submit their responses once again. The stepwise procedure is given below:

Step 1: Respondents provide pairwise comparison of dimensions and variables. The ranking scale is depicted using a scale of 1 to 9 , where 1 indicates same preference and 9 represents the intense preference of dimensions, and variables. Meanwhile, ranking 3, 5, and 7 indicate little, strong and very strong preference. Based on the given input by the respondents, an $n \times n$ reciprocal matrix has been conducted where $n$ represents dimensions of HRIS adoption. Sample input for the respondent and the matrix has been given in Fig. 1. The dimensions are represented in a short form such as Org, Hum, and Tech, Env for organization, human, technology and environment respectively. All elements in the reciprocal matrix should be greater than zero, i.e $\mathrm{a}_{\mathrm{ij}}>0$ where $\mathrm{a}_{\mathrm{ij}}$ represents an element inside the matrix of row $\mathrm{i}$ and column $\mathrm{j}$.

Step 2: Sum up each and every column of the reciprocal matrix. Divide every component of the matrix with the summation of its column. As a result, normalized relative weight matrix has been obtained. Whenever an average of the rows is being done, we can find the normalized principal 
Eigenvector or priority vector. The priority vector can be depicted by multiplying by 100 to get the percentage of preference of the elements.

Step 3: Find out the consistency (CI) of the respondents: $\mathrm{CI}=\frac{\lambda \max -\mathrm{n}}{\mathrm{n}-1}$ where $\lambda_{\max }$ is the principal Eigen value determined by summing up products between each element of Eigen vector and summation of columns of reciprocal matrix.

$\lambda_{\max }$ can be computed using the following formula:

$\sum \mathrm{w}_{\mathrm{i}} \mathrm{x}_{\mathrm{j}}$ where $\mathrm{x}_{\mathrm{j}}=$ sum of column of comparison matrix and $\mathrm{w}_{\mathrm{i}}=$ priority vector , here $\mathrm{i}=\mathrm{j}=\mathrm{n}$.

Step 4: If $\mathrm{n}>2$, calculate consistency ratio (CR) using CI and Random consistency index (RCI) proposed by Prof Satty given solution using 500 matrices as sample size. The formula of $\mathrm{CR}$ is given as below:

$C R=\frac{C I}{R I}$

Step 5: If $\mathrm{CR}<10 \%$, the judgment is considered to be consistent, otherwise the respondent was requested to reconsider preferences once again.

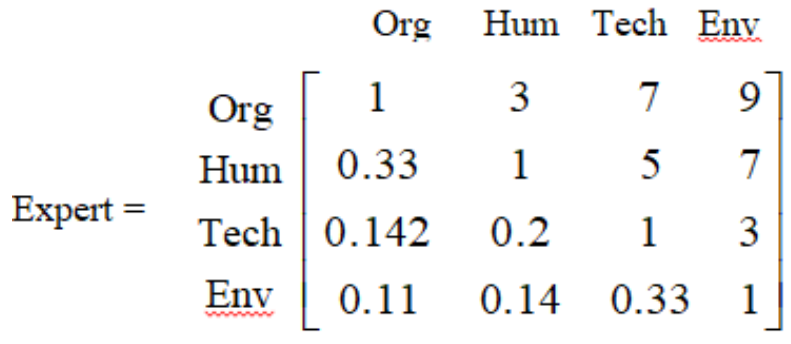

Fig. 1. Sample Matrix for four Dimensions Given by the Expert11.

TABLE I. USING AHP TO FIND OUT THE RANKING OF DIMENSIONS

\begin{tabular}{|l|l|l|l|l|}
\hline Experts & Technology & Organization & Environment & Human \\
\hline Expert 1 & 9.02 & 57.39 & 4.44 & 29.13 \\
\hline Expert 2 & 8.39 & 65.77 & 5.17 & 20.66 \\
\hline Expert 3 & 10.78 & 56.28 & 6.21 & 26.71 \\
\hline Expert 4 & 11.03 & 52.54 & 6.33 & 30.08 \\
\hline Expert 5 & 12.18 & 55.78 & 5.68 & 26.33 \\
\hline Expert 6 & 12.18 & 55.78 & 5.68 & 26.33 \\
\hline Expert 7 & 9.99 & 67.15 & 5.98 & 16.85 \\
\hline Expert 8 & 12.18 & 55.78 & 5.68 & 26.33 \\
\hline Expert 9 & 9.02 & 57.39 & 4.44 & 29.13 \\
\hline Expert 10 & 8.39 & 65.77 & 5.17 & 20.66 \\
\hline Expert 11 & 10.78 & 56.28 & 6.21 & 26.71 \\
\hline Expert 12 & 11.03 & 52.54 & 6.33 & 30.08 \\
\hline Expert 13 & 12.18 & 55.78 & 5.68 & 26.33 \\
\hline Expert 14 & 12.18 & 55.78 & 5.68 & 26.33 \\
\hline Expert 15 & 9.02 & 57.39 & 4.44 & 29.13 \\
\hline Sum of score & 158.44 & 867.48 & 83.21 & 390.85 \\
\hline Average & $10.56 \%$ & $57.83 \%$ & $5.54 \%$ & $26.05 \%$ \\
\hline Rank & 3 & 1 & 4 & 2 \\
\hline
\end{tabular}

respondents (Expert $1 \ldots$ Expert 15) and find out the highest value to be ranked 1 and so on for dimensions and respective variables in Table I. Thus, the highest and lowest priority of the dimensions as well as variables can be found out. The rankings are represented from higher ranking to lower ranking. It can be clearly seen that the organization dimension receives the highest average value (57.83\%) whereas the environment receives the lowest average value $(5.54 \%)$. Thus, organization is ranked as the most preferred one (ranked $1^{\text {st }}$ ) whereas environment is the least preferred one (ranked 4th) among the respondents. Moreover, human is ranked at 2nd with $26.05 \%$ and technology is ranked at 3rd with $10.56 \%$.

\section{B. Decision Making Trail Evaluation Laboratory (DEMATEL)}

DEMATEL algorithm has been used in the next section in order to find out the most important causes as well as the correlations among variables. It reveals the influence between the variables. The steps have been described as follows:

Step 1: Respondents will provide the influence based on the factors (dimensions and variables). The influence will be based on the assumptions given in step 1 of Section 3.1. Based on the ranking, answer matrices (let's name it as $\mathrm{x}$ ) will be constructed. If there are $\mathrm{H}$ respondents and $\mathrm{n}$ factors, then there will be $\mathrm{H}$ matrices each comprised of $\mathrm{n}$ factors. Thus there will be $\mathrm{H}$ matrices each composed of $\mathrm{n} \times \mathrm{n}$ factors.

Step 2: Construct an initial direct relation matrix. It is also known as average influence qualification. Each and every component will be measured as $\mathrm{a}_{\mathrm{ij}}=\frac{\sum X_{i j} \mathrm{H}}{\mathrm{n}}$ where $\mathrm{i}$ and $\mathrm{j}$ represent row and column of answer matrix and $n$ is the number of factors. Sum up all the components of each and every row and take the largest sum of the row as G. Now the value of $G$ will be used to generate normalized direct relation matrix (D). $G=\max _{1 \leq i \leq n} \sum_{j=1}^{n} a_{i j}$

Step 3: Direct relation matrix's Normalized form $\mathrm{D}$ will be computed by dividing all the elements of $\mathrm{A}$ by $\mathrm{G}$.

Step 4: Total relation matrix will be worked out using the following formula: $\mathrm{T}=\mathrm{D}$ (I-D)-1. The total relation matrix of the four variables has been shown below.

Step 5: (special condition): If $\mathrm{i}==\mathrm{j}$, then, $\mathrm{ri}+\mathrm{cj}$ indicates the total effect given and received by factor $\mathrm{i}$ and $\mathrm{ri}-\mathrm{cj}$ indicates net effect factor $i$ has on the system.

If $\mathrm{ri}-\mathrm{cj}>0$, factor $\mathrm{i}$ is a causer, else Factor $\mathrm{i}$ is a receiver, it is also known as effect.

The Total relation matrix for the Variables of Technology dimension is:

\begin{tabular}{|c|c|c|c|c|c|c|c|}
\hline \multirow{4}{*}{ Tech $=$} & V5 & 0.1072 & 0.1913 & 0.0687 & 0.0278 & 1.1936 & -0.4036 \\
\hline & $\begin{array}{l}\text { V6 } \\
\text { V7 }\end{array}$ & 0.0646 & 0.1064 & 0.0266 & 0.0164 & 1.6767 & -1.2487 \\
\hline & V8 & 0.1879 & 0.4559 & 0.1074 & 0.0708 & 1.2031 & 0.4409 \\
\hline & V9 & 0.4389 & 0.7091 & 0.1783 & 0.1066 & 1.6543 & 1.2113 \\
\hline
\end{tabular}


Where, V1=COM_ADV, V2= PER_COMPA, V3= PER_COMPL, V4= IT_INFRAS

The Total relation matrix for the Variables of Organization dimension is:

Org $=$\begin{tabular}{cccccccc}
\multicolumn{2}{c}{ V5 V6 V7 V8 V9 $\mathrm{r}_{\mathrm{i}}+\mathrm{c}_{\mathrm{i}} \mathrm{r}_{\mathrm{i}}-\mathrm{c}_{\mathrm{i}}$} \\
V5 & 0.0899 & 0.6443 & 0.4056 & 0.1565 & 0.2379 & 1.7515 & 1.3168 \\
V6 & 0.0136 & 0.0899 & 0.0515 & 0.0197 & 0.0183 & 2.0489 & -1.6629 \\
V7 & 0.0220 & 0.1679 & 0.0897 & 0.0244 & 0.0486 & 1.4268 & -0.7215 \\
V8 & 0.0545 & 0.4502 & 0.3518 & 0.0895 & 0.1590 & 1.4501 & 0.7599 \\
V9 & 0.0373 & 0.5037 & 0.1757 & 0.0549 & 0.0898 & 1.4152 & 0.3078
\end{tabular}

Where, V5= TLS, V6= OC, V7= OST, V8= OSIZE V9= PER_COST

The Total relation matrix for the Variables of Human dimension is:

$$
\begin{aligned}
& \text { V10 V11 V12 } r_{i}+c_{j} r_{i}-c_{i} \\
& \begin{array}{rllllll}
\text { Hum }= & \text { V10 } & 0.1745 & 0.0839 & 0.4764 & 1.3845 & 0.0851 \\
\text { V11 } & 0.4013 & 0.1742 & 0.9675 & 1.8370 & 1.2489 \\
\text { V12 } & 0.0738 & 0.0359 & 0.1755 & 1.9045 & -1.3340
\end{array}
\end{aligned}
$$

Where, V10=CHAR_SEC, V11=SIS, V12=EMPB_CHAR

The Total relation matrix for the Variables of environment dimension is:

$$
\begin{aligned}
& \text { V13 V14 V15 } r_{i}+c_{j} r_{i}-c_{j} \\
& \text { Env }=\begin{array}{llllll}
\text { V13 } & 0.1326 & 0.4735 & 0.8160 & 1.6171 & 1.2272 \\
\text { V14 } & 0.0402 & 0.1328 & 0.3137 & 1.1518 & -0.1783 \\
\text { V15 } & 0.0222 & 0.0587 & 0.1320 & 1.4747 & -1.0489
\end{array}
\end{aligned}
$$

Where, V13=CP, V14=TVS, V15=GOV_SUP

\section{Net Effect and Total Effect of Variables}

In the technology dimension, the value of $\mathrm{r}-\mathrm{c}$ is greater than 0 for V3 and V4. More specifically, the value of perceived complexity and IT infrastructure are 0.4409 and 1.2113. So, these are the major causes of the technology dimension and should not be overlooked. The higher value of $r+c$ indicates higher prominence. The Value of $r+c$ is highest for variable $\mathrm{V} 2$, namely perceived compatibility, revealing the fact that this variable has major interaction with other variables compared to the interaction of other variables with this variable.

Same rule applies for organization, human and environment dimensions' variables. It can be unequivocally seen in the organization dimension that variable V5, top level support is the most important cause while variable V6, organizational culture has the strongest correlation than any other criteria for the organizational dimension. The variable V4, IT infrastructure is the most important cause in the technological dimension. Variable V11, Staffs IT skill is the most significant cause that has impact on other variables whereas V12, employee behavioral characteristics have major correlations with all other factors in the system in terms of human dimension. Turning to dimension environment, the variable competitive pressure is the significant causer affecting other variables.
In nutshell, top level support is the most crucial factor in the organization dimension and Staffs IT skill is the most significant in terms of human dimension. Moreover, IT infrastructure is the most vital factor in the technology dimension. Turning to environment dimension, variable competitive pressure has been considered to be having the prominent significance.

\section{Discussions}

This research adds new ideas to existing HRIS literature. The investigation explores the impacting variables on decision making for adopting HRIS in the industries of Bangladesh. The exploration of factors based on the theoretical model overcomes the limitations explained in the HOT-fit model and the TOE model. Analyzing the respondents' opinion, the research explored that organization is ranked as the most important one (ranked first) whereas environment is the least important one (ranked fourth). Moreover, human is ranked at second and technology dimension is ranked at third.

In human dimension, IT expertise of staff is recognized as the most significant driver that inducing HRIS usage in the organizations. This outcome is consistent with past studies [5, $12,33]$. In order to continue consistent growth and keeping long term focus in business organizations, the availability of expert HRIS is a vibrant factor in the IT-dependent HRM applications. In a contemporary study, researchers quantified that personnel who have both technical and managerial skills are considered as the most valuable asset for a firm [25]. It also shows the importance of technology readiness for any technological acceptance. So, HR professionals should be enriched with multidisciplinary knowledge of application based IT and the functions of HR to ensuring effective HRIS operations in organizations. However, this result rejects some contemporary studies where IT expertise of staff was identified as an insignificant driver for IT innovation adoption $[2,13]$.

In organization dimension, this exploration finds that the top level support is factually critical determiner for HRIS utilization. In this way, administration support from higher authority is vital for distribution of recourse and to motivate people to use the system. The finding also supports past studies $[2,4,5,12]$. So, active support of top management speeds up the HRIS adoption in the organizations. Furthermore, to success the adoption project, top management provides sufficient resources such as people, materials, capital, and related support. The outcome of this study indicates that adopting a new technology in organizations will be easier to the organizations when the senior executives support the innovation adoption. Moreover, sometimes, innovation adoption encounter barrier within the organizations. At that time, top management helps to overcome these problems. So, this study strongly recognized the importance of top level support that significantly influences the incumbents to use HRIS in routine HRM tasks Conversely, this result rejects some contemporary studies where top level support was identified as an non-significant driver for HRIS implementation and usage [11, 14].

In technology dimension, the outcome demonstrates that IT infrastructure is positioned as highest -ranked factor to HRIS selection in the organizations of Bangladesh. Also, this variable 
was discovered to have huge impact in earlier research on IT application selection [34, 35]. Likewise, Masum [36] additionally uncovered IT infrastructure as a critical success factor in organizations of Bangladesh. The contemporary research shows that it progressively becomes important to ensure that the HRIS fits in with the existing IT infrastructure for information systems used in an organization. Also, HRIS modules need easy to understand and user friendly interface. In almost all developing countries, related costs to IT, necessary infrastructure, and the quality of these infrastructures hinders the adoption of IT applications in the organizations. Nevertheless, this result rejects some current studies where IT infrastructure was acknowledged as an insignificant factor for IT innovation adoption [5, 21].

From environment dimension, competitive pressure is signified to be dominant drivers for HRIS use in organizations. It implied that organizations are feeling pressure for using new technologies such as HRIS to gain competitive advantage and to achieve its goals .The results of previous research support the finding of the current study [14, 37, 38]. So, this study suggests that organization should adopt proper strategies to handle the competitive pressure as; nowadays, new technology adoption crying need of survival in the industry. In earlier research, researchers stated that a company cannot perceive competitive advantage without properly managing their human resource and IT applications [25]. Currently, organizations are adopting HRIS to support in getting maximum results from their employees, making better-informed decisions and streamlining HR processes and better distribution of human resources. Therefore, these things encourage other organizations to adopt and use the HRIS as well as related IT applications are being competitive in the holistic spectrum of business. However, the present research confronts findings of Ahmad, Abu Bakar, Faziharudean and Mohamad Zaki [39], Ahmadi, Nilashi and Ibrahim [11], and Teo, Lim and Fedric [4]. The researchers stated that competitive pressure is an insignificant factor for HRIS adoption and usage.

\section{CONCLUSIONS}

This paper combines two theories of adoption such as HOT-fit model and TOE framework to recognize the factors influencing the organizations of emerging country like Bangladesh to implement and usage of HRIS for managing human resources efficiently and effectively. The findings of the research will enhance the managers to identify the issues that are related to adoption, implementation, and usage. As far our knowledge goes, organization-level adoption behavior of HRIS using MCDM techniques has never been examined in Bangladesh. Thus, this paper added value to the HRM discipline by improving present understanding of HRIS adoption issues, which is an unsearched field in Bangladesh. This study only applied 15 determinants to inspect the decision of use HRIS. Importantly, some others relevant factors may also influence HRIS usage such as pressure from trading partners, data security, government policy, and information intensity etc. For further research, the findings of the study might be used as a generalized model for the developing countries to take decision adopting HRIS in the organizations.

\section{REFERENCES}

[1] K. M. Masum, "Adoption Factors of Electronic Human Resource Management (e-HRM) in Banking Industry of Bangladesh," Journal of Social Sciences, vol. 11, no. 1, pp. 1, 2015.

[2] R. H. Al-Dmour, "An integration model for identifying the determinants of the adoption and implementation level of HRIS applications and Its effectiveness in business organisations in Jordan ", Computer Science, Brunel University London, UK, 2014.

[3] A. S. Narayana, and R. L. Bhusal, "Adoption and Use of Human Information System Digital Technology for Organizational Competitiveness: An Exploratory Study in the Context of Nepal," Handbook of Research on Social and Organizational Dynamics in the Digital Era, pp. 250-275: IGI Global, 2020.

[4] T. S. Teo, G. S. Lim, and S. A. Fedric, "The adoption and diffusion of human resources information systems in Singapore," Asia Pacific Journal of Human Resources, vol. 45, no. 1, pp. 44-62, 2007.

[5] M. G. R. Alam, A. K. M. Masum, L.-S. Beh, and C. S. Hong, "Critical Factors Influencing Decision to Adopt Human Resource Information System (HRIS) in Hospitals," PloS one, vol. 11, no. 8, pp. e0160366, 2016.

[6] A. Davarpanah, and N. Mohamed, "Human Resources Information Systems Implementation and Influences in Higher Education: Evidence From Malaysia," International Journal of Asian Business and Information Management (IJABIM), vol. 11, no. 3, pp. 65-84, 2020.

[7] A. K. M. Masum, L.-S. Beh, M. A. K. Azad, and K. Hoque, "Intelligent human resource information system (i-HRIS): a holistic decision support framework for HR excellence," Int. Arab J. Inf. Technol., vol. 15, no. 1, pp. 121-130, 2018.

[8] T. Bondarouk, E. Parry, and E. Furtmueller, "Electronic HRM: four decades of research on adoption and consequences," The International Journal of Human Resource Management, vol. 28, no. 1, pp. 98-131, 2017.

[9] C. R. Greer, Strategic human resource management, 2001.

[10] Y. Alshamaila, S. Papagiannidis, and F. Li, "Cloud computing adoption by SMEs in the north east of England: A multi-perspective framework," Journal of enterprise information management, vol. 26, no. 3, pp. 250$275,2013$.

[11] H. Ahmadi, M. Nilashi, and O. Ibrahim, "Organizational decision to adopt hospital information system: An empirical investigation in the case of Malaysian public hospitals," International journal of medical informatics, vol. 84, no. 3, pp. 166-188, 2015.

[12] L. Bian, “An Empirical Study on Factors that Influencing the Adoption of Electronic Human Resource Management (E-HRM) Among Firms in Northeast of China," University of Malaya, 2012.

[13] C. Low, Y. Chen, and M. Wu, "Understanding the determinants of cloud computing adoption," Industrial management \& data systems, vol. 111, no. 7, pp. 1006-1023, 2011.

[14] A. Gutierrez, E. Boukrami, and R. Lumsden, "Technological, organisational and environmental factors influencing managers' decision to adopt cloud computing in the UK," Journal of Enterprise Information Management, vol. 28, no. 6, pp. 788-807, 2015.

[15] S. Jackson, "Organizational culture and information systems adoption: A three-perspective approach," Information and Organization, vol. 21, no. 2, pp. 57-83, 2011.

[16] Y.-M. Wang, Y.-S. Wang, and Y.-F. Yang, "Understanding the determinants of RFID adoption in the manufacturing industry," Technological forecasting and social change, vol. 77, no. 5, pp. 803-815, 2010.

[17] D. R. Cooper, and P. Schindler, Business research methods, 8th ed., Irwin, Boston: McGraw-Hill, 2003.

[18] T. S. Teo, S. Lin, and K.-h. Lai, "Adopters and non-adopters of eprocurement in Singapore: An empirical study," Omega, vol. 37, no. 5, pp. 972-987, 2009.

[19] M. Ghobakhloo, D. Arias-Aranda, and J. Benitez-Amado, "Adoption of e-commerce applications in SMEs," Industrial Management \& Data Systems, vol. 111, no. 8, pp. 1238-1269, 2011. 
[20] O. Mothobi, and L. Grzybowski, "Infrastructure deficiencies and adoption of mobile money in Sub-Saharan Africa," Information Economics and Policy, vol. 40, pp. 71-79, 2017.

[21] G. Kannabiran, and P. Dharmalingam, "Enablers and inhibitors of advanced information technologies adoption by SMEs," Journal of Enterprise Information Management, 2012.

[22] R. Rahayu, and J. Day, "Determinant Factors of E-commerce Adoption by SMEs in Developing Country: Evidence from Indonesia," ProcediaSocial and Behavioral Sciences, vol. 195, pp. 142-150, 2015.

[23] I. Troshani, C. Jerram, and S. R. Hill, "Exploring the public sector adoption of HRIS,” Industrial Management \& Data Systems, 2011.

[24] A. K. M. Masum, M. J. Kabir, and M. M. Chowdhury, "Determinants that influencing the adoption of E-HRM: An empirical study on Bangladesh," Asian Social Science, vol. 11, no. 21, pp. 117, 2015.

[25] A. K. M. Masum, M. G. R. Alam, M. S. Alam, and M. A. K. Azad, "Adopting factors of electronic human resource management: Evidence from Bangladesh." pp. 1-4.

[26] M. S. Amalnick, A. Ansarinejad, and S.-M. Nargesi, "New perspective to ERP critical success factors: Priorities and causal relations under fuzzy environment," Journal of Mathematics and Computer Science, vol. 2, pp. 160-170, 2011.

[27] Q. N. Naveed, M. R. N. Qureshi, N. Tairan, A. Mohammad, A. Shaikh, A. O. Alsayed, A. Shah, and F. M. Alotaibi, "Evaluating critical success factors in implementing E-learning system using multi-criteria decisionmaking," Plos one, vol. 15, no. 5, pp. e0231465, 2020.

[28] S. Rouhani, A. Ashrafi, and S. Afshari, "Segmenting critical success factors for ERP implementation using an integrated fuzzy AHP and fuzzy DEMATEL approach,” World Applied Sciences Journal, vol. 22, no. 8, pp. 1066-1079, 2013.

[29] J.-C. Pomerol, and S. Barba-Romero, Multicriterion decision in management: principles and practice: Springer Science \& Business Media, 2012.

[30] C.-Y. Huang, J. Z. Shyu, and G.-H. Tzeng, "Reconfiguring the innovation policy portfolios for Taiwan's SIP Mall industry," Technovation, vol. 27, no. 12, pp. 744-765, 2007.
[31] F.-Y. Pai, "Analyzing consumers' decisions to select micro-invasive aesthetic service providers using a hybrid method," Applied Mathematics \& Information Sciences, vol. 8, no. 6, pp. 3071, 2014.

[32] J.-S. Horng, C.-H. Liu, S.-F. Chou, Y.-S. Yin, and C.-Y. Tsai, "Developing a novel hybrid model for industrial environment analysis: A study of the gourmet and tourism industry in Taiwan," Asia Pacific Journal of Tourism Research, vol. 19, no. 9, pp. 1044-1069, 2014.

[33] A. K. M. Masum, M. J. Kabir, and M. M. Chowdhury, "Determinants that Influencing the Adoption of E-HRM: An Empirical Study on Bangladesh," Asian Social Science, vol. 11, no. 21, pp. p117, 2015.

[34] H. Sulaiman, and N. Wickramasinghe, "Assimilating healthcare information systems in a Malaysian Hospital," Communications of the Association for Information Systems, vol. 34, no. 1, pp. 66, 2014.

[35] W.-H. Hung, I. Chang, D. C. Yen, and C.-M. Lee, "Critical Factors of Adopting Enterprise Application Integration Technology: An Empirical Study on Larger Hospitals," Communications of the Association for Information Systems, vol. 36, no. 1, pp. 31, 2015.

[36] A. K. M. Masum, "Adoption Factors of Electronic Human Resource Management (e-HRM) in Banking Industry of Bangladesh," Journal of Social Sciences/Sosyal Bilimler Dergisi, vol. 11, no. 1, 2015.

[37] N. Al-Qirim, "The adoption of eCommerce communications and applications technologies in small businesses in New Zealand," Electronic Commerce Research and Applications, vol. 6, no. 4, pp. 462473, 2008.

[38] B. Ramdani, D. Chevers, and D. A. Williams, "SMEs' adoption of enterprise applications: A technology-organisation-environment model," Journal of Small Business and Enterprise Development, vol. 20, no. 4, pp. 735-753, 2013.

[39] S. Z. Ahmad, A. R. Abu Bakar, T. M. Faziharudean, and K. A. Mohamad Zaki, "An Empirical Study of Factors Affecting e-Commerce Adoption among Small-and Medium-Sized Enterprises in a Developing Country: Evidence from Malaysia," Information Technology for Development, vol. 21 , no. 4, pp. 555-572, 2014. 